\title{
MASE-BDI: Agents with Practical Reasoning for Land Use and Cover Change Simulation
}

\author{
Carolina G. Abreu ${ }^{1}$, Cássio G. C. Coelho ${ }^{1}$, Célia G. Ralha ${ }^{1}$ \\ ${ }^{1}$ Department of Computer Science - University of Brasília (UnB) \\ Brasília - DF - Brazil \\ carolabreu@unb.br, \{cassio,ghedini\}@cic.unb.br
}

\begin{abstract}
Agents with a cognitive dimension are paramount to represent and understand land use and land cover changes that involves decision making. A Belief-Desire-Intention(BDI)-Agent system for environmental simulation was developed:the MASE-BDI framework. MASE-BDI, a novel version of MASE, implements agents that can be represented by their individual beliefs and intentional behavior to choose plans of action in a complex environment. We investigate the advantages, limitations and drawbacks of this new design and how practical reasoning agents can contribute to decision support for sustainability. Experiments were made in a spatially explicit LUCC study case of the Brazilian Cerrado between the years of 2002 and 2008. MASE-BDI simulation results were compared to those obtained with the multi-agent system for landuse change simulation previously developed in this research project.
\end{abstract}

\section{Introduction}

To manage the land use and land cover changes (LUCC) is likely to be the most significant challenge facing the Earth over the next decades. The effects of LUCC extend land surface and affects the Earth's climate, hydrology and ecological processes. Over the next century, global population is projected to increase by $50-100 \%$ and it is likely that there will also be an increase of the pressures to further convert or manage "natural" ecosystems for human needs. LUCC research aims to support insightful management of land resources in order to avoid irreversible damage [Le et al. 2008].

Understanding the patterns of LUCC has increased significantly over the last decade [Turner II 2002]. This has been facilitated in part by the increased awareness of the issues and by the large number of focused studies directed to understanding the underlying driving forces for LUCC dynamics. But there is still much debate when it comes to aiming for environmental sustainability while ensuring or maintaining economic development. Recent researches reinforced the role of information technology on improving environmental sustainability in terms of information, representation, organization, innovative strategies and evaluation of systems that break new ground in environmental responsibility [Elliot 2011]. Thus, policy makers and governmental planners in the LUCC sector start to rely on software, such as simulators, to do analysis of land resources and prepare the stakeholder dialogue on LUCC management decisions [Kangas and Kangas 2005]. Multi-agent system (MAS) is a well-suited approach that can be used in simulators to help to answer some environmental research-relevant questions.

MAS aims to reproduce the knowledge and reasoning of several heterogeneous agents that need to coordinate their actions to jointly solve planning problems through 
time. Much research has been made on MAS because it offers an abstraction mechanism to allow researchers to manage complexity with greater ease and therefore overcome difficulties that the complex nature of LUCC imposes on traditional modeling approaches, such as statistical modeling, transition probabilities or classical optimization [Verburg 2006]. MAS allows to incorporate the influence of human decision making on land use, along with the appropriate environmental feedbacks, in a formal and spatially explicit way, taking into account social interaction, adaptation and decision making at different levels [Matthews et al. 2007].

The MultiAgent System for Environmental Simulation (MASE) is a computational system that uses a configurable user model to examine and to simulate the human impacts of changing land use and land management in both spatially and temporally explicit manner [Ralha et al. 2013a]. MASE ${ }^{1}$ aims to assist analyzing LUCC dynamics using technical information to aid the decision making process. It effectively illustrates the environmental, economic and social dynamics while driven by regional management policies. Although MASE was proven a valid solution, ecologists still strive for new methodologies to represent the human action, the environment and its relations. Most MAS architectures include reactive agents, nevertheless, when human actions are considered in the ecosystem, architectures place more emphasis on deliberation. Nonetheless, a cognitive dimension is primordial for all ecological systems [Bousquet and Le Page 2004]. One common approach adopted in the Artificial Intelligence (AI) field is to build agents as intentional systems. One of the objectives of this research project is to investigate if those AI cognition techniques could help solve environmental problems.

We present an extended version of MASE with a high-level agent architecture that implements a Belief-Desire-Intention (BDI) model. MASE-BDI adopts agents with individual knowledge bases and intentional behavior to represent the complex, dynamic and error-prone LUCC environment. We refactored all MASE code regarding the agents: its internal logic, architecture, communication and interaction protocol, and the way agents perceive and act in the environment. The LUCC model used in MASE-BDI is the same as the one used in MASE, and we tested it in the same case study, to establish a comparison. Although the use of a BDI architecture is foretell to be a promising method, it is not yet critically reviewed or broadly adopted in the LUCC scientific community. Thus, the present research goal is to investigate the advantages, limitations and drawbacks of applying cognitive agents into the MASE framework. This paper is organized as follows: Section 2 discuss the MASE-BDI design and presents some implementation details. Section 3 reports on the LUCC study case, a set of experiments in a Cerrado area of the Brazilian Federal District, and Section 4 present the results. Section 5 summarizes the findings and suggests future research issues.

\section{THE MASE-BDI}

In ecosystems, BDI architectures were first applied in [Bousquet et al. 1993]. However, most applications for ecological problems use simple agents and attention focuses on understanding their coordination or their relations with the environment. Although authors recognize the importance of the cognitive to represent the complexity of agents in the environmental models, the general rule for ecological applications is the absence of

\footnotetext{
${ }^{1}$ http://mase.cic.unb.br/
} 
it[Railsback et al. 2006]. The MASE-BDI framework was designed to meet this lack of cognitive tools. Its reasoning engine is based on the BDI model, where agents can be represented by their individual beliefs and intentional behavior to choose plans of action in a complex environment [Bratman 1987][Dennett 1989].

MASE-BDI is an hybrid MAS that enables modeling and simulations of LUCC dynamics using a configurable user model with both top-down and bottom-up structures simultaneously [Verburg 2006]. For comparison purposes, MASE-BDI uses the same methodological two-fold approach used to define MASE: (i) the systematic and structured empirical characterization of the model [Smajgl et al. 2011]; and (ii) the conceptual structure definition according to the agent-based model documentation protocol - Overview, Design concepts and Details [Grimm et al. 2006]. The adopted conceptual structure is adequate for conducting experiments which tests, for example, human understanding of key processes in land-use changes and other environmental issues. See [Ralha et al. 2013a] for a complete description of the MASE System.

The MASE novel approach implements a BDI agent architecture design, adopting agents with individual knowledge bases and intentional behavior. We investigate the advantages, limitations and drawbacks of this new design in a spatially explicit LUCC study case of the Cerrado area of the Brazilian Federal District between the years 2002 and 2008. The role of the cognitive agents is evaluated. The results were compared to those obtained with MASE previous architecture. The following sections present the architecture, execution model and implementation aspects of MASE-BDI, highlighting the key features that set MASE and MASE-BDI apart.

\subsection{Architecture Overview}

MASE-BDI was built upon the JADEX agent framework, since we used the JADE platform to develop MASE and wanted to keep the developed functionalities [Pokahr et al. 2003]. JADE is a middleware that garantees the FIPA-related apliance with issues such interoperability, security, and maintainability. JADEX is an extension of JADE that provides both a middleware and a reasoning-oriented system. The reasoningoriented feature emphasizes on rationality and goal-directedness, with a reasoning engine based on the BDI model. To access functionality of the JADEX system, a Java API is provided for basic actions such as sending messages, manipulating beliefs or creating subgoals.

The MASE-BDI high level architecture is composed of three hierarchical layers: from the top, an User Interface (UI) layer, a Pre-processing layer and an Agent layer. The UI layer is composed by a graphical interface, which enables the visualization of the simulation, and a JADEX module, the JADEX Control Center (JCC). The Pre-processing layer groups a set of objects responsible for the image processing of the simulation and the configuration of the user model. Those images and data are set by the user. These two layers are responsible for the support of the Agent Layer, the practical implementation of the BDI model. This layer contains a set of agents that interacts with one another. Each agent is coupled with an individual Belief Database and all of them makes use of the same Resoning Engine.

The MASE-BDI Agent Classes were named after the main abstractions of a LUCC environment: GRID Manager, Spatial Manager, Transformation Manager and Transfor- 
mation Agents. The internal structure and reasoning mechanisms are the same for each class of agents. From the outside, an agent is perceived as a black box, which receives and sends messages. Incoming messages, as well as internal events and new goals serve as input to the agent's internal reaction and deliberation mechanism. Based on the results of the deliberation process these events are dispatched to already running plans, or to new plans instantiated from the plan library. Running plans may access and modify the belief base, send messages to other agents, create new top-level or subgoals and cause internal events.

The reaction and deliberation mechanisms are generally the same for all agents. The behavior of a specific agent is therefore determined solely by its concrete beliefs, goals, and plans, set by the user in the conceptual model. The MASE-BDI characterization follows the guideline provided by JADEX [Pokahr et al. 2003]. The beliefs are represented by Java Objects contained in the belief base as named facts that can be directly manipulated by setting, adding or removing facts. The beliefs are used as input for reasoning engine by specifying certain belief states, e. g. as preconditions for plans.

Goals are concrete, momentary desires of an agent. A deliberation mechanism is responsible for managing the state transitions of all goals. As restricted by JADEX, a sophisticated goal deliberation mechanism is not yet available, therefore currently the JADEX engine automatically activates all valid goal options. In MASE-BDI, a perform goal is directly related to the execution of actions. Therefore the goal is considered to be reached, when some actions have been executed, regardless of the outcome of these actions. In a LUCC metaphor this can mean that a transformation agent of the agricultural type may have a goal to explore the land until there are still natural resources to be exploited. For as long there are trees to cut, this agent will be reaching its goal.

The implemented reasoning engine handles all event such as the reception of a message or the activation of a goal by selecting and executing appropriate plans. Instead of performing ad-hoc planning, MASE-BDI uses the plan-library approach defined by JADEX to represent the plans for each agent. For each plan, a plan head defines the circumstances under which the plan may be selected and, a plan body specifies the actions to be executed. The plan bodies, which are predefined courses of actions are implemented as Java classes. JADEX provides a basic Java API for basic action such as sending messages, manipulating beliefs, or creating goals.

The agents are created based on a LUCC conceptual model. To create and start an agent, the system needs to know the properties of the agent to be instantiated. The initial state of an agent is determined among other things by the beliefs, goals and the library of known plans. The plan bodies are implemented as Java classes. All other concepts are specified in a so called agent definition file (ADF) using a XML language that follows the JADEX framework. A predefined package of functionality related entities (beliefs, goals and plans) can be clustered and it is called an agent capability. Agents can have multiple capabilities.

This internal structure will work together as the simulation goes on. Each step of the simulation will be executed following an integration protocol and an execution model. The following section will present the overall mechanisms to understand the MASE-BDI reasoning engine. 


\subsection{Execution Model and Interaction Protocol}

Figure 1 illustrates the components of the MASE-BDI reasoning engine and its relations. The functional elements of the execution model follow the BDI realization plan. Some plans, internal events and goals of a Transformation Agent, i.e. a rancher in a LUCC simulation, are detailed in Figure 1 but the structure is the same for each class of agents. The core of any BDI architecture is the mechanism of plan selection: an agent has to receive information from the environment (messages or internal events), choose the plan that best fit its goals, execute the plan and keep track of the plan steps to notice failures. In the MASE-BDI, all of the required functionality is implemented in separated components. The relevant information about beliefs, goals and plans is stored in data structures accessible to all the components of the reasoning engine.

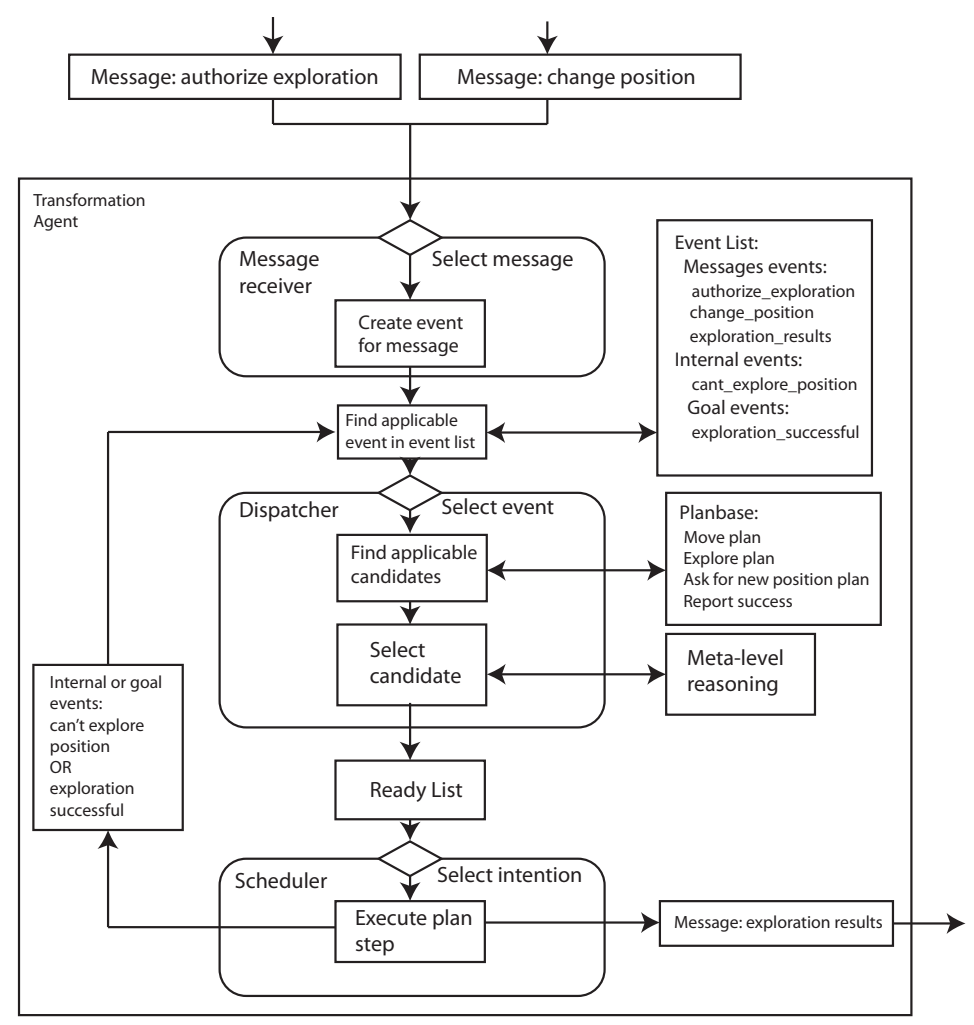

Figure 1. Overview of the MASE-BDI Execution Model

The Message Receiver performs operations that take FIPA ACL messages from another agent and create internal or external events that are placed in an event list. The Dispatcher selects events and builds a list of applicable plans. It also selects the plans to be executed from the Plan base, in a deliberation step, associating each plan to the corresponding event or goal. The Scheduler takes each plan, set by the Dispatcher as ready, and executes them. As a consequence of the execution of a plan new messages or events will be created and the belief database may be altered.

The simulation is made by the interaction of all class of agents. The interaction protocol is FIPA compliant and the basic unit of communication is a message. Figure 2 shows some of the main messages exchanged in a LUCC simulation. Through time, this messages set the course of action of the simulation. The basic land use exploration is the 


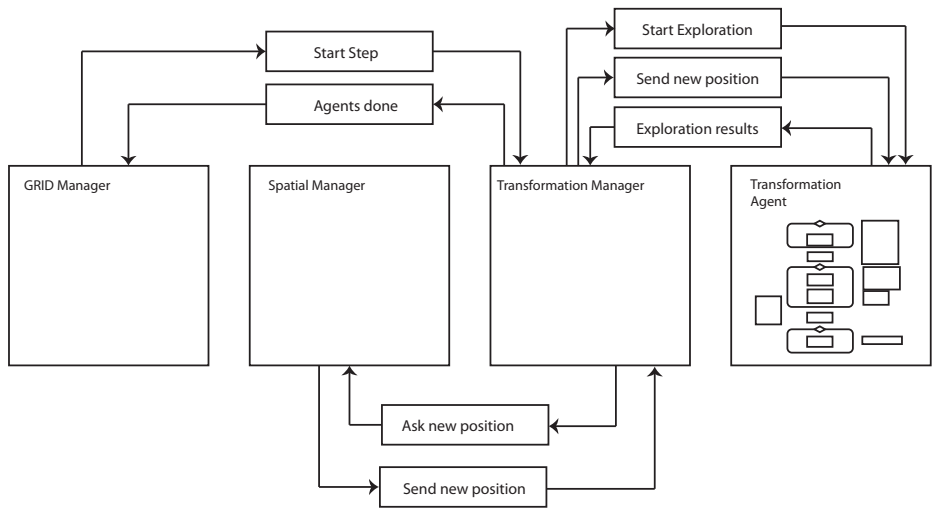

Figure 2. Overview of the MASE-BDI Interaction Protocol

outcome of the workflow presented in this Figure. The GRID Manager (GRIDM) starts the simulation running a step. The Transformation Manager (TM) perceives this event and send multiple messages to all Transformation Agents (TA), authorizing the exploration to start or the TA to move around the space. The TA will execute its plans of land use, exploring and moving around the environment. When it's done, it returns the exploration results to the TM. If the plan execution of TA fails, due i.e. to a land exhaustion or because there is another TA exploring the intended exploration space, the plan is dropped and the TM is informed of the conflict. Thenceforth, the TM requests to the Spatial Manager (SM) anther available land space and informs the TA its new position. The TA then updates its belief, restarts and wait for a new chance to be deployed and explore its new space. This cycle will continue until the goals of the simulation are done.

\section{The Cerrado Case Study}

The land use/cover change of Brazil's Federal District (DF) Cerrado was chosen as a case study in MASE and therefore was maintained for comparison reasons in MASE-BDI. The Cerrado is a Brazilian savanna-like ecosystem and is the second largest biome in Brazil, occupying approximately $22 \%$ of the country surface area. The Cerrado is one of the most threatened biomes of the world, mainly due to the rapid agriculture expansion [Sano et al. 2008]. The input of the simulation used two land cover maps provided by the Brazilian Institute of Environment and Renewable Resources (IBAMA) at a initial time (2002 - t0) and a subsequent time (2008 - t6). Those maps are public domain data and are used by the government in the deforestation control and land use planning.

As in the MASE experiments, the simulation was performed in steps, where each step corresponds to a week time in real life. The total area of study was divided into cells, in which every set of four cells represents one hectare, which is occupied by a different agent. The physical state of the cells correspond to the set of real spatial data including six proximal variables: (i) water courses (rivers); (ii) water bodies (lakes); (iii) buildings; (iv) highways; (v) streets; and (vi) protected areas. In this first experiment using cognitive agents the human factor over the land is represented by two types of transformation agents: farmers and ranchers. The political aspects are also taken as a compelling force in the simulation, translating the Federal District Spatial Plane (PDOT) onto an influence matrix for the transformation agents. In both simulations the proximal variables are used in order to create a probabilist model that reflects the likelihood of 
exploration.

We performed both MASE and MASE-BDI simulations using identical parameters to compare the results found in both agent architectures. The MASE-BDI simulations were performed using 10 to 100 transformation agents. In each simulation, the last step was saved in an image, named simulated 2008, so it could be compared with the image representation of observed 2008 and a score would be obtained. Moreover, the image representation of the observed 2002 was compared with the observed 2008 using the same method of the simulated images comparison, described in [Pontius et al. 2008]. The validation is based on a quantitative agreement with the observed changes, and we calculate the sum of the LUCC area and the pixel by pixel comparison, which includes the allocation of land cover change. Those metrics are the ones often used in the benchmarking of LUCC modelling tools, as presented by [Paegelow et al. 2014]. The null model threshold is then obtained, a representation of a simulation with zero agents. This measurement is a reference score for all simulation, as theirs score should be better than this borderline score.

\section{Experimental Results and Discussion}

The baseline MASE results are the ones presented in [Ralha et al. 2013b]. The MASEBDI simulation results are illustrated in Figures 3 and 4. Figure 3 presents the DF area and the changes and persistence in the land cover affected by anthropic processes and natural vegetation. The real observed change and the MASE predicted change are put aside for comparison. Figure 4 shows us a three map comparison using (i) a reference map of initial time (2002), (ii) a reference map of subsequent time (2008), and (iii) a MASE prediction map of the subsequent time (2008). According to the methodology, the three-map comparison specify the amount of the predictions accuracy that is attributable to land persistence versus land change. It is possible to pinpoint the locations where the simulation was accurate and the locations where the MASE predicted land cover changes different from the observed.

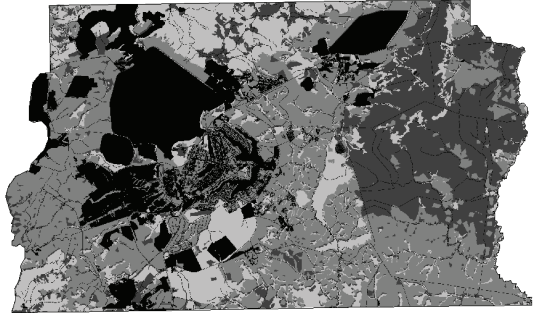

a. Observed

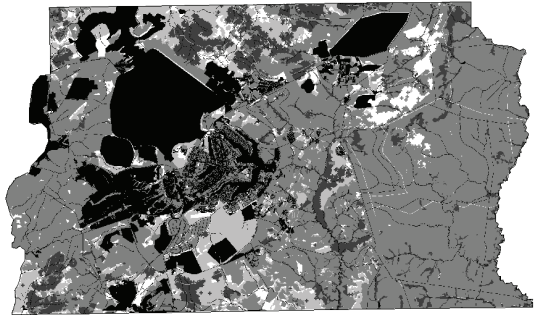

b. Predicted
Layers

Anthropic gain Anthropic persistence Native vegetation persistence

Figure 3. MASE-BDI model predictions for the Brazilian Federal District, showing (a) Observed change 2002-2008, (b) Predicted change 2002-2008

Different applications can be summarized and compared using two statistics: the null model resolution (NMR) and the figure of merit(FoM). The simulation results indicate the potential of the presented multi-agent model system. Considering the accuracy of the simulations using MASE-BDI, the application results were better than the null model, that examines both the behavior of the model and the dynamics of the landscape. The definition of this null model is a prediction of complete persistence, i.e. no change, 


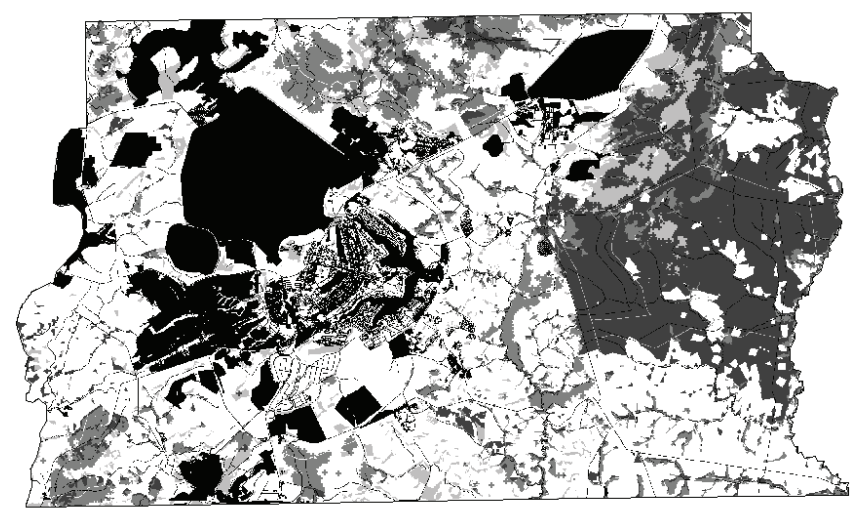

Correct due to observed change predicted as change

Error due to observed change predicted as persistence

Error due to observed persistence predicted as change

Correct due to observed persistence predicted as persistence

Figure 4. Three map comparison: the final image comparing (i) a reference map of initial time (2002), (ii) a reference map of subsequent time (2008), and (iii) a MASE-BDI prediction map of the subsequent time (2008)

between the initial and the subsequent time, therefore the accuracy of the null model is $100 \%$ minus the amount of observed change.

Considering the figure of merit, the more accurate applications are the ones where the amount of observed net change in the reference maps is larger. The figure of merit is the ratio of the intersection of the observed change and predicted change to the union of the observed change and predicted change, which can range from $0 \%$ (no overlap between observed and predicted change) to $100 \%$ (perfect overlap between observed and predicted change, a perfect accurate prediction). MASE-BDI Figure of Merit best results were found in the simulations with 80 and 90 agents, where the FoM reached 55\%. In MASE previous results, the maximum value of FoM were $54 \%$ in a simulation with 90 agents. The FoM best values in MASE-BDI varied from $51 \%$ to $55 \%$ while in MASE those values were $50 \%$ to $53 \%$. These prediction measures may be seen as too low to those who are used to purely statistical models, but according to [Pontius et al. 2008], LUCC frameworks that are able to reach a FoM higher than $50 \%$ are exceptionally rare.

\section{Conclusions}

This article presents MASE-BDI, an extended version of MASE with a high-level agent architecture that implements BDI. The BDI design and the agents execution model were presented and a case study was performed. The results shows us that even with a more complex cognitive agent architecture, MASE-BDI was able to surpass MASE previous results in the same case study. Even though MASE-BDI is a more refined solution it was designed to be flexible to the user, with real gain in modularity, clarity and reuse of the models.

MAS provide an efficient tool to assess ecological complexity and to assist ecosystem management. The MASE-BDI prototype allows the user to investigate researchrelevant questions such as how effective are management practices, such as ecologicaleconomic zoning, or how effective are deliberate changes in land use, such as reforestation on LUCC dynamics. Moreover, the basic components of a system designed for a 
dynamic, uncertain world should include some representation of beliefs, desires, intentions and plans. The notions of complexity and change, inherent to ecological problems, have a major impact on the way the computational systems are built. We believe that software agents, in particular BDI agents, can provide the essential components necessary to cope with the real world complex scenarios.

We have proposed a BDI approach to simulate the human decision making process in a LUCC dynamic environment. This approach is particularly useful for applications where human decision affect directly the land use planning process. The user may create different types of planning for agents in the simulation. MASE framework will held the mechanisms for those agents to select the plan according to the environment and to execute the currently active plan during the hole simulation process. As future work we intend to integrate additional transformation agents to demonstrate the system's activities coordinating capability considering a large number of agents. To evaluate the use of the cognitive agents in MASE-BDI it is necessary a comprehensive assessment of errors and uncertainties in the model simulation. Future work will include a detailed error analysis and uncertainty treatment, possibly a undecidability model for the simulations will be proposed.

\section{References}

Bousquet, F., Cambier, C., Mullon, C., Morand, P., Quensiere, J., and Pavé, A. (1993). Simulating the interaction between a society and a renewable resource. Journal of Biological Systems, 01(02):199-214.

Bousquet, F. and Le Page, C. (2004). Multi-agent simulations and ecosystem management : a review. Ecological Modelling, 176:313-332.

Bratman, M. (1987). Intention, Plans, and Practical Reason. Harvard University Press, Cambridge, Massachusetts.

Dennett, D. C. (1989). The Intentional Stance. A Bradford book. MIT Press, Cambridge, Massachusetts.

Elliot, S. (2011). Transdisciplinary perspectives on environmental sustainability: A resource base and framework for it-enabled business transformation. Management Information Systems Quarterly, 35(1):197-236.

Grimm, V., Berger, U., Bastiansen, F., Eliassen, S., Ginot, V., Giske, J., Goss-Custard, J., Grand, T., Heinz, S. K., and Huse, G. (2006). A standard protocol for describing individual-based and agent-based models. Ecological Modelling, 198(1-2):115-126.

Kangas, J. and Kangas, A. (2005). Multiple criteria decision support in forest management - the approach, methods applied, and experiences gained. Forest Ecology and Management, 207(1-2):133-143.

Le, Q. B., Park, S. J., Vlek, P. L., and Zambonelli, F. (2008). Land-use dynamic simulator (LUDAS): A multi-agent system model for simulating spatio-temporal dynamics of coupled human landscape system. Structure and theoretical specification. Ecological Informatics, 3(2):135-153. 
Matthews, R. B., Gilbert, N. G., Roach, A., Polhill, J. G., and Gotts, N. M. (2007). Agentbased land-use models : a review of applications. Landscape Ecology, pages 14471459.

Paegelow, M., Camacho Olmedo, M. T., Mas, J.-F., and Houet, T. (2014). Benchmarking of LUCC modelling tools by various validation techniques and error analysis. Cybergeo, 701(online):29.

Pokahr, A., Braubach, L., and Lamersdorf, W. (2003). Jadex: Implementing a BDIInfrastructure for JADE Agents. EXP, 3(3):76-85.

Pontius, RobertGilmore, J., Boersma, W., Castella, J.-C., Clarke, K., Nijs, T., Dietzel, C., Duan, Z., Fotsing, E., Goldstein, N., Kok, K., Koomen, E., Lippitt, C., McConnell, W., Mohd Sood, A., Pijanowski, B., Pithadia, S., Sweeney, S., Trung, T., Veldkamp, A., and Verburg, P. (2008). Comparing the input, output, and validation maps for several models of land change. The Annals of Regional Science, 42(1):11-37.

Railsback, S. F., Lytinen, S. L., and Jackson, S. K. (2006). Agent-based simulation platforms: Review and development recommendations. SIMULATION, 82(9):609-623.

Ralha, C. G., Abreu, C. G., Coelho, C. G. C., Zaghetto, A., Macchiavello, B., and Machado, R. B. (2013a). A multi-agent model system for land-use change simulation. Environmental Modelling \& Software, 42(0):30 - 46.

Ralha, C. G., Abreu, C. G., and Coelho, C. G. D. C. (2013b). Mase : Multi-agent system for environmental simulation. In IV Workshop de Computação Aplicada à Gestão do Meio Ambiente e Recursos Naturais, pages 1-5, Maceio-AL. SBC.

Sano, E. E., Rosa, R., Brito, J. L. S., and Ferreira, L. G. (2008). Mapeamento semidetalhado do uso da terra do bioma cerrado. Pesquisa Agropecuaria Brasileira, 43(1):153156.

Smajgl, A., Brown, D. G., Valbuena, D., and Huigen, M. G. A. (2011). Empirical characterisation of agent behaviours in socio-ecological systems. Enviromental Modelling \& Software, 26(7):837-844.

Turner II, B. L. (2002). Toward integrated land-change science advances in 1.5 decades of sustained international research. In Steffan, W., Jager, J., Carson, D., and Bradshaw, C., editors, Challenges of a Changing Earth: Proceedings of the Global Change Open Science Conference, Amsterdam, NL, 10-13 July 2000, pages 269-272. Springer Verlag.

Verburg, P. H. (2006). Simulating feedbacks in land use and land cover change models. Landscape Ecology, 21(8):1171-1183. 